\title{
Survey of pre-clerkship medical students' knowledge and perceptions of anesthesiology at one Canadian university
}

\author{
Emilie M. Robertson, BSc $\cdot$ Sarah J. Henschke, BSc • \\ Jay Friesen, MA $\cdot$ Ban C. H. Tsui, MD
}

Received: 27 October 2014/Accepted: 9 December 2014/Published online: 16 December 2014

(c) Canadian Anesthesiologists' Society 2014

\section{To the Editor,}

Several published surveys have suggested that medical students generally lack sufficient knowledge of the scope of anesthesiology practice. ${ }^{1-3}$ Despite this, anesthesiology is one of the most highly competitive specialties among graduates seeking residency positions in Canada. In 2014, it ranked as the fifth highest first-preference specialty among Canadian medical graduates while offering only $3.5 \%$ of the total residency positions. ${ }^{4}$ In 2014 , only $73 \%$ of applicants were matched to anesthesiology as their first-choice discipline, a decrease from $77 \%$ the previous year. ${ }^{5}$ This paradox suggests that sometime between pre-clerkship and application for residency a number of Canadian medical students gain sufficient awareness and knowledge of anesthesiology to be interested in pursuing it as a career. To collect information about pre-clerkship students' attitudes, knowledge, and perception of anesthesiology, we surveyed first- and second-year medical students at the University of Alberta ( $\mathrm{U}$ of A), whose medical school has no in-class units designated to teach anesthesiology during the students' pre-clerkship.

Electronic supplementary material The online version of this article (doi:10.1007/s12630-014-0297-4) contains supplementary material, which is available to authorized users.

E. M. Robertson, BSc $\cdot$ S. J. Henschke, BSc .

B. C. H. Tsui, MD (ه)

Department of Anesthesiology and Pain Medicine, University

of Alberta, Edmonton, AB, Canada

e-mail: btsui@ualberta.ca

J. Friesen, MA

Department of Modern Languages and Cultural Studies,

University of Alberta, Edmonton, AB, Canada
The survey (see Appendix; available as Electronic Supplementary Material) consisted of 14 questions designed to collect information about students' knowledge of anesthesiology practice and their perceptions of anesthesiologists' job satisfaction, financial compensation, and lifestyle. Surveys were distributed to the 165 first-year and 168 second-year students. Responses were received from 137 (83\%) and 132 (79\%) students, respectively. Figure A shows the first- and second-year students' primary source of information about anesthesiology. Students' perceptions of an anesthesiologist's role, financial compensation, and workload are shown in Figure B. Basic knowledge of where anesthesiologists work in Canada varied dramatically, from being highly accurate in certain areas to lacking considerably in others. Overall, $94 \%$ of students reported interest in learning more about anesthesiology (45\% very interested, $49 \%$ somewhat interested), whereas $6 \%$ did not answer or reported no interest. When asked in what year they would be most interested in learning about anesthesiology, the most frequent response was the second year (39\%) followed by the third year (28\%). Figure $\mathrm{C}$ shows that shadowing and mandatory rotation were almost equally chosen (94 and 90 responses, respectively) as the preferred methods to learn more about anesthesiology.

Our survey reveals that pre-clerkship students at the $\mathrm{U}$ of A medical school possess reasonable general knowledge about anesthesiology. They also have overall positive perceptions of anesthesiologists' financial compensation and relative workload. A vast majority of the students reported interest in learning more about anesthesiology. Our survey also yielded some concerning results. For example, whereas no first-year students reported no interest in learning more about anesthesiology, $8 \%$ of second-year students gave this answer (data not shown). One possibility 
Figure A) Primary sources about anesthesiology among first- and second-year medical students. B) Students' perceptions of anesthesiologists' role, financial compensation, and workload. C) Students' preferred methods of learning about anesthesiology

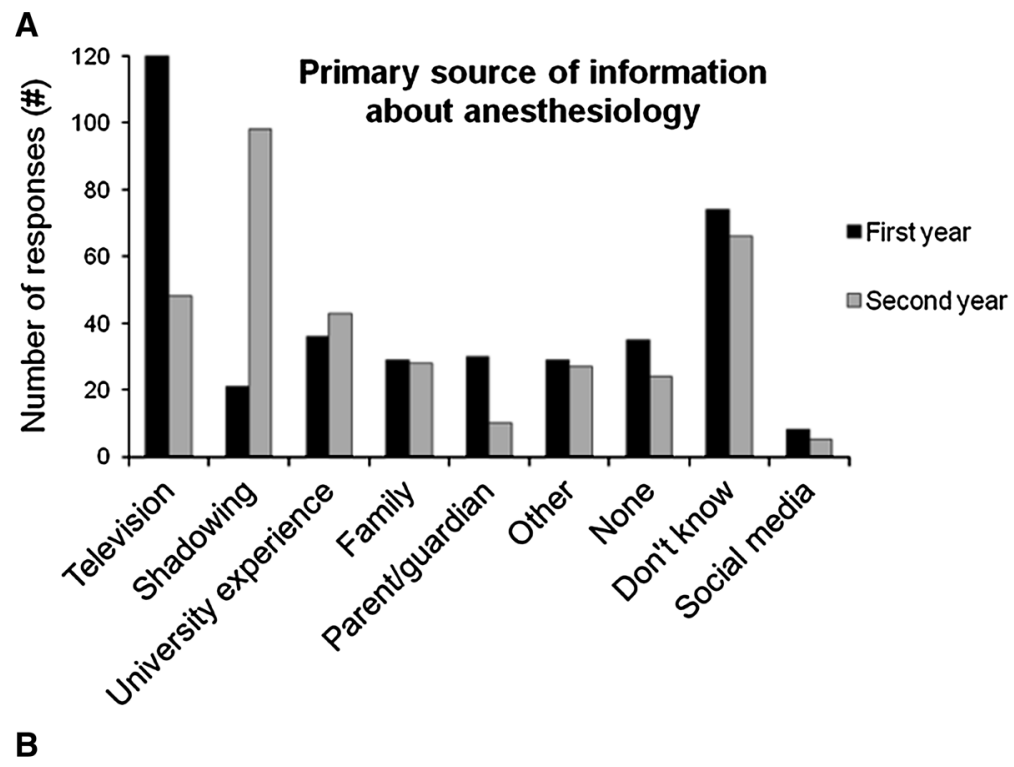

"An anesthesiologist's role is more like a technician than a physician" 18 32 50

"An anesthesiologist is overpaid for the work they do compared to other medical specialists" 17

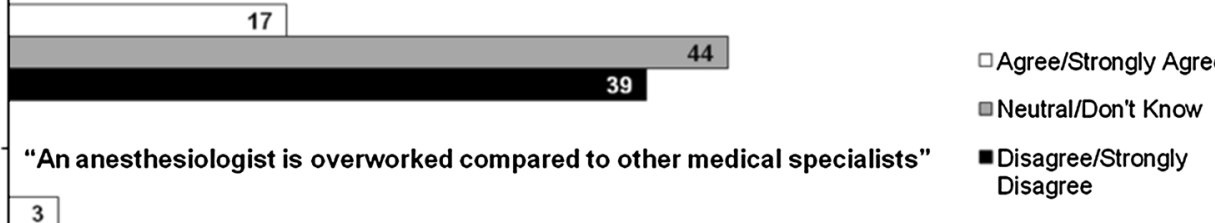
3 53 44

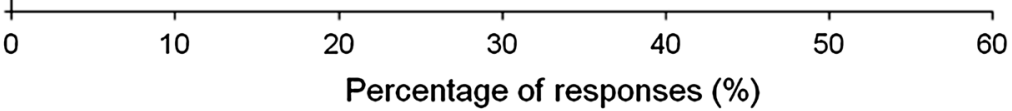

C

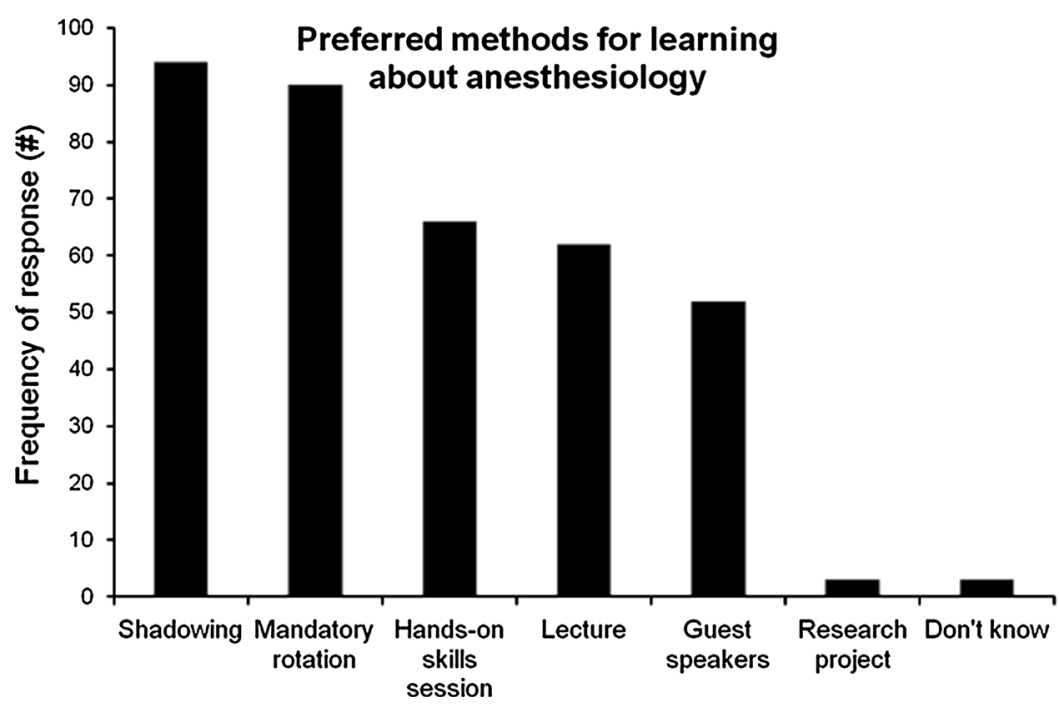


is that some second-year students might have had a negative first-hand experience(s) with anesthesiology during shadowing or with a faculty interaction. The fact that more second-year students obtained their knowledge of anesthesiology through shadowing than did first-year students (Figure A) suggests that shadowing experiences may be responsible for negatively influencing second-year students' perception of the specialty. This is particularly relevant at the $U$ of $A$, which offers no in-class anesthesiology teaching during the pre-clerkship. The absence of a mandatory anesthesiology rotation during clerkship also means that uninterested students lack a potential opportunity to gain a positive experience or to form a favourable perception of anesthesiology.

This survey does have some limitations. First, it was conducted at a single institution. Second, the survey was not fully externally validated, although we used questions/ statements that two independent research consultants determined were not leading or confusing. Nevertheless, our study provides a good foundation for examining factors that influence medical students' choice of anesthesiology as a career path. We intend to survey the same cohort of students as they complete their clerkship to determine if, how, and why their attitudes and perceptions of anesthesiology have changed during their undergraduate medical education.

Acknowledgements The authors thank Dr. Andrea WernerLeonard for assistance in designing the questionnaire. The authors also thank Drs. Gary Hnatko and Sarah Forgie and the students for participating in the study and completing the questionnaires. Dr. Tsui has been given a Clinical Scholar Award from the Alberta Heritage Foundation for Medical Research (AHFMR). Dr. Tsui's research is supported by the Canadian Anesthesia Research Foundation.

Conflicts of interest None declared.

\section{References}

1. DeMaria S Jr, Bryson EO, Bodian C, et al. The influence of simulation-based physiology labs taught by anesthesiologists on the attitudes of first-year medical students towards anesthesiology. Middle East J Anaesthesiol 2011; 21: 347-53.

2. Levin KJ, Friedman $C P$, Scott PV. Anesthesiology and the graduating medical student: a national survey. Anesth Analg 1979; 58: 201-7.

3. Smith A, Mannion S, Iohom G. Irish Medical Students Knowledge and Perception of Anaesthesia. Education in Medicine Journal, North America, June 2013. Available from URL: http://www. eduimed.com/index.php/eimj/article/view/144 (accessed December 2014).

4. Canadian Resident Matching Service (CaRMS). 2014 R-1 Main Residency Match Report (June 2014). Available online at: http:// www.carms.ca/en/data-and-reports/r-1/reports-2014/ (accessed December 2014).

5. The Canadian Federation of Medical Students. The Match Book: An annual review of the residency match by the Canadian Federation of Medical Students 2014-2015. Available from URL: http://www.cfms.org/resources-3/publications/cfms-annual-review. html (accessed December 2014). 